\title{
Fuzzy and Position Particle Swarm Optimized Routing in VANET
}

\author{
Viswanathan Ramasamy \\ Kongu Engineering College, \\ Department of Mathematics, \\ Erode, Tamil Nadu, India \\ Visu.maths1@gmail.com \\ Jagatheswari Srirangan \\ Vellore Institute of Technology, \\ Department of Mathematics, \\ Vellore, Tamil Nadu, India \\ jaga.sripa@gmail.com
}

\author{
Praveen Ramalingam \\ Anna University, \\ Department of Computer Science and Engineering, \\ Chennai, Tamil Nadu, India \\ Praveenram2510@gmail.com
}

\begin{abstract}
In Intelligent Transport Systems, traffic management and providing stable routing paths between vehicles using vehicular ad hoc networks (VANET's) is critical. Lots of research and several routing techniques providing a long path lifetime have been presented to resolve this issue. However, the routing algorithms suffer excessive overhead or collisions when solving complex optimization problems. In order to improve the routing efficiency and performance in the existing schemes, a Position Particle Swarm Optimization based on Fuzzy Logic (PPSO-FL) method is presented for VANET that provides a high-quality path for communication between nodes. The PPSO-FL has two main steps. The first step is selecting candidate nodes through collectively coordinated metrics using the fuzzy logic technique, improving packet delivery fraction, and minimizing end-to-end delay. The second step is the construction of an optimized routing model. The optimized routing model establishes an optimal route through the candidate nodes using position-based particle swarm optimization. The proposed work is simulated using an NS2 simulator. Simulation results demonstrate that the method outperforms the standard routing algorithms in packet delivery fraction, end-to-end delay and execution time for routing in VANET scenarios.
\end{abstract}

Keywords: VANET, Position Particle Swarm Optimization, Fuzzy Logic, coordinated metrics, candidate nodes

\section{INTRODUCTION}

VANETs have recently attracted attention for their ability to enable inter-vehicle communications. As a result, efficient routing is one of the methods for improving vehicular traffic safety. Ad hoc On-demand Distance Vector adaptation in the Virtual Node layer (VNAODV) [1] improves packet delivery fraction and latency. However, with so many messages to send, so much overhead, and so many collisions, the network lifetime was reduced. [4] proposes FB QoS-Vanet, a QoS-based routing protocol that accommodates applications with QoS requirements. Long Lifetime Anypath (LLA) [16] was created to address connection link stability, ensuring stable communication pathways to reduce overhead and extend the network's life. Clustering and cluster-based routing techniques were described in [2] to obtain a higher delivery ratio and much lower overhead for delay-tolerant networks. In [7], a novel polynomial-time sequential topology inference algorithm was designed to reduce probing overhead and handle node dynamics.

According to recent experiments, multi-path fading and co-channel interference influence packet delivery in wireless mesh networks. [9] proposed a Cross-Layer Channel Adaptive Routing protocol to improve channel and transmission rate. Aside from significant routes, path maintenance and latency were also considered, which identified and corrected the point of failure before performing recovery action. However, increased scalability posed severe threats to energy consump- 
tion. To address energy consumption issues, [21] designed an energy-aware clustering algorithm that addressed energy consumption and improved network lifetime. The method described in [15] is applicable in dynamic network conditions and compatible with both vehicle-to-infrastructure and vehicle-to-vehicle communication modes. The authors of [3] designed a bi-objective optimization formulation to analyze linkstability to ensure energy-aware routing in distributed wireless networks. [5] developed a stochastic model with a density-dependent velocity profile for optimizing both transport and communication networks. [14] proposed interference-aware routing based on passive measurements to improve routing efficiency. In [10], the authors investigated some traditional features of the particle swarm in search of properties.

We propose Position Particle Swarm Optimization with Fuzzy Logic for vehicular ad hoc networks with correlated metrics, the cores of a Collective Coordinated Fuzzy Logic algorithm and a Position-based Particle Swarm Optimization algorithm in this paper. The Collective Coordinated Fuzzy Logic algorithm selects candidate nodes by considering correlated metrics, bandwidth, node energy, and mobility to improve packet delivery fraction in VANET. A position-based particle swarm optimization algorithm is proposed to reduce the execution time during routing, which improves the efficiency of routing in VANET by eliminating the inertial weight and control coefficients in PSO. As a result, it can enhance VANET routing efficiency and extend network lifetime.

The remainder of the paper is structured as follows: section 2 reviews some related works on routing algorithms. section 3 suggests using fuzzy logic to optimize position particle swarms, and section 4 contains a detailed description of the simulation results as well as discussions. Finally, section 5 brings the work to a close.

\section{RELATED WORKS}

The works of literature related to link correlation and data acquisition concerning routing are described in this section. A novel link correlation aware opportunistic routing scheme was designed in [18] by applying link correlation metrics. In [13], a framework for secure and data acquisition methods was presented using advanced encryption standard. Another acknowledgement-based broadcast protocol was investigated in [17] using connected dominating sets to provide higher reliability and message delivery.

Recently, VANETs have emerged as one of the most demanding research areas, ensuring safety in Intelligent Transport system networks. In [8], a Trajectorybased Statistical Forwarding scheme was presented in the road network that ensured data forwarding and reduced the packet delivery delay fraction through an optimal rendezvous point. However, with the increasing number of vehicles, the routing overhead also increased. To reduce the routing overhead, In [22], a neighbour coverage-based probabilistic rebroadcast protocol was designed aiming at reducing the number of retransmissions. In [6], a machine learning-based adaptive routing protocol was developed based on reinforcement learning for underwater sensor networks.

Geographic routing is attractive since the routing state needed for greedy forwarding at each node is highly based on the network size. In [11], a geographic routing protocol for d-dimensional spaces was investigated to reduce the node churn rate. In [20], opportunistic routing was considered to estimate the traffic flow density based on the road traffic flow and geographic topology. Robust routing and scheduling mechanisms designed in [19] in dynamic conditions aim to improve routing efficiency. Another method to address reliable packet delivery using beacon distance vector-based global routing and distance vector-based local routing was designed in [12] to achieve scalability and efficiency in a wide range of scenarios.

\section{POSITION PARTICLE SWARM OPTIMIZATION WITH FUZZY LOGIC(PPSO-FL)}

The proposed PPSO-FL focuses on enhancing the routing operations in VANETs, with the amalgamation of collectively coordinated metrics and position-based particle swarm optimization. In this section, a routing operation is described by starting with a network model, followed by Fuzzy Logic-based Candidate node Selection, and finally, ends with an optimal route through Position-based Particle Swarm Optimization.

\subsection{NETWORK MODEL}

Let us consider a unidirectional network of length ' $l$ '. For vehicles' $V=v_{1}, v_{2}, \ldots, v_{n}{ }^{\prime}$ on the network, there occurs ' $D$ ' discrete speed levels with speed ' $v$ ' seen by the observer as ' $\lambda_{i}$. Therefore, the overall vehicle arrival rate is expressed as given below:

$$
\operatorname{VAR}=\sum_{\mathrm{i}=1}^{\mathrm{n}} \lambda_{\mathrm{i}}
$$

With the above vehicle arrival rate 'VAR', the probability of occurrence of speed ' $v_{i}^{\prime}$ is expressed as given below:

$$
\operatorname{Prob}_{i}=\frac{\lambda_{i}}{V A R}
$$

Let us now model a network topology in the form of a graph' $G=(V, E)$ '. Here, vehicles (referred to as nodes) represent vertices set ' $V=\left\{V_{i}\right\}^{\prime}$ and links to the set of time-dependent edges ' $E=\left\{e_{i j}\right\}$ ' with every node having the same transmission radius ' $R$ '. An edge ' $e_{i j}$ ' is said to exist if the distance 'Dist ${ }_{i j}$ ' between two nodes' $v_{i}^{\prime}$ and ' $v$ ' is less than or equal to ' $R$ ' and is expressed as given below:

$$
E=\left\{e_{i j} \mid v_{i}, v_{j} \in V, D_{i s t} t_{i j} \leq R\right\}
$$

The problem lies in designing an optimized routing method elaborated in the forthcoming subsections with the above network model. 


\subsection{FUZZY BASED CANDIDATE SELECTION}

The PPSO-FL uses collectively coordinated metrics, including bandwidth, node energy, and mobility of the node, to identify the feasible links between the nodes using the candidates. The efficient nodes from the set of nodes in VANET are selected as candidates. This selection is made based on the improved bandwidth ' $B E^{\prime}$ rate, possessing high energy ' $E(T)$ ' and low mobility ' $D R_{i j}$ ' pattern using a fuzzy logic model. Fig. 1 shows a simple fuzzy logic model for candidate node selection.

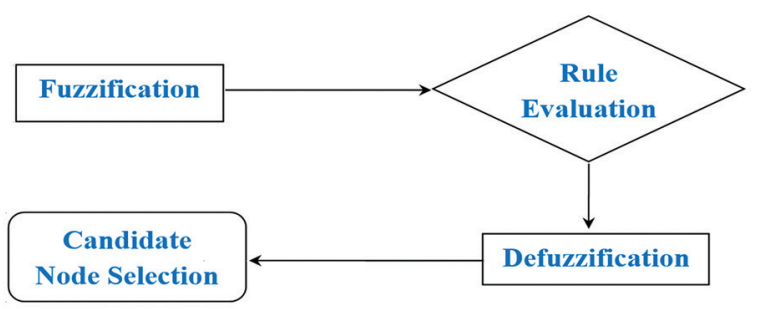

Fig. 1 Candidate node selection using the fuzzy logic

Once the network is deployed with the nodes, each node broadcasts a 'HELLO' packet to its neighbour nodes. The broadcasted packet includes the source node identifier ' $v_{i}$ ', Destination node ' $v_{n}$ ' and the collective coordinated metrics bandwidth ' $v(B)$ ', node energy ' $v(E)$ ' and mobility of the node ' $v(M)$ ' respectively, as shown in Fig. 2.

\begin{tabular}{|l|l|l|l|l|}
\hline$v_{i}$ & $v(B)$ & $v(E)$ & $v(M)$ & $v_{n}$ \\
\hline
\end{tabular}

Fig. 2 Format of hello message

The candidate nodes are ascertained with the aid of the fuzzy logic model. Fig. 3 shows the process of Fuzzy Logic-based Candidate node Selection.

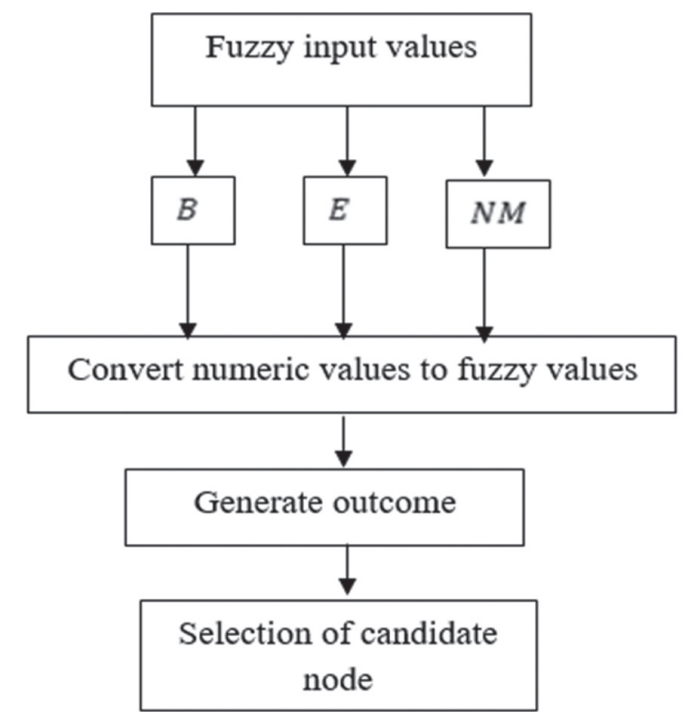

Fig. 3 Process of Fuzzy Logic-based Candidate node Selection.
As shown in Fig. 3, metrics bandwidth ' $v(B)$ ', node energy ' $v(E)$ ', and mobility of the node ' $v(M)$ ' are given as the input to the fuzzy model. Whenever a source node in the network needs to transfer message packets to the destination node, the PPSO-FL evaluates the local bandwidth ' $B$ ' based on the transmission range of neighbours ' $R\left(v_{i}\right)$. Thus, the node monitors the transmission channel, measures the bandwidth estimation, and is expressed below:

$$
B E=T C C * \frac{T_{\text {idle }}}{T_{\text {tot }}}
$$

From (4), the bandwidth ' $B E$ ' is measured based on the product of transmission channel capacity 'TCC' and the ratio of the idle time ' $T_{\text {idle }}$ ' to the overall time ' $T_{\text {tot }}$ '. Once the bandwidth is estimated, the node energy is evaluated. Let us consider a node with initial energy as ' $E_{i}$ ' then, the energy consumed over a period of time ' $T$ ' is expressed as given below:

$$
E(T)=D P_{t}+D P_{r}
$$

From (5), the energy consumed ' $E(T)$ ' is the summation of the number of data packets transmitted, ' $D P_{t}$ ' and received ' $D P_{r}^{\prime}$ ' over some time ' $T$. Finally, the node mobility of ' $v_{i}^{\prime}$ concerning node ' $v_{j}^{\prime}$ is evaluated based on the distance rate ' $D R_{i j}$ ' and is expressed as given below:

$$
D R_{i j}=\frac{\sqrt{\left(v_{i}-v_{b}\right)^{2}+\left(v_{b}-v_{j}\right)^{2}}}{R}
$$

Where ' $\left(v_{i}-v_{b}\right)$ ' and ' $\left(v_{b}-v_{j}\right)$ ' are the Cartesian products of nodes ' $v_{i}$ ' and ' $v_{j}^{\prime}$ ' respectively with ' $R$ ' corresponding to the transmission range. The fuzzification converts the numeric values to fuzzy values from the above-evaluated bandwidth rate (6), energy, and node mobility. Followed by this, the membership functions are evaluated as expressed below:

$$
M F\left(F u z z y_{i}\right) \rightarrow M A X(B E), M A X(E(T)), M I N\left(D R_{i j}\right)
$$

With the membership function measured using (7), the rule evaluation with higher bandwidth, node possessing high energy and low mobility is expressed below:

$$
\begin{aligned}
& f(\text { outcome })= \\
& \left\{\begin{array}{c}
\text { low, bandwidth is high, energy is high } \\
\text { and node mobility is low } \\
\text { high, bandwidth is low, energy is low } \\
\text { and node mobility is high }
\end{array}\right.
\end{aligned}
$$

The interpretation of the above rule is that higher bandwidth, energy, and low node mobility are a desirable selection which yields a high collective coordinated outcome. Finally, the PPSO-FL produces a numeric result based on the output membership function called defuzzification and is expressed below:

$$
D=\frac{F u z z y_{i} * M F\left(F u z z y_{i}\right)}{M F\left(F u z z y_{i}\right)}
$$

From (8), a node with the maximum collective coordinated outcome is selected as the candidate node. Fig. 4 shows the Collective Coordinated Fuzzy Logic algorithm. 
Input: Nodes' $V=v_{1}, v_{2}, \ldots, v_{n}{ }^{\prime}$, Node Mobility' $M^{\prime}$, Bandwidth' $B$ ', Energy' $E^{\prime}$, Candidate Node ' $C N=$ $c n_{1}, c n_{2}, \ldots, c n_{n}{ }^{\prime}$

\section{Output: Optimal Routing}

\section{1: Begin}

2: For each HELLO message broadcasted by nodes' $V^{\prime}$ with input values' $B$ ', ' $E$ ' and ' $M$ ' to neighboring nodes

3: $\quad$ Measure Bandwidth Estimation using (4)

4: $\quad$ Measure Energy consumed over a while ' $T$ ' using

(5)

5: $\quad$ Measure node mobility using (6)

6: $\quad$ Evaluate membership functions using (7)

7: $\quad$ Generate the outcome based on the results of (5), (6), and (7)

8: If bandwidth is high, energy is high, and node mobility is low

9: $\quad$ Then the outcome is low

10: Node is selected as candidate node ' $C N$ '

11: $\quad$ Else outcome is high

12: $\quad$ Go to step 2

\section{3: $\quad$ End if}

\section{4: End for}

\section{5: End}

Fig. 4 Collective Coordinated Fuzzy Logic algorithm

As shown in Fig.4, the Collective Coordinated Fuzzy Logic algorithm uses fuzzy logic for the candidate node selection. If the source node needs to send a data packet to the destination node in the network, the PPSO-FL initially calls the fuzzy logic that selects the candidate nodes through which the data packets are sent in VANET. In the PPSO-FL, the candidate nodes are selected using the Collected Coordinated metrics, namely, Bandwidth estimation, Node energy, and node mobility. The nodes with high bandwidth, high energy, and low mobility are selected as the candidate node through which the data packets are sent. As a result, the packet delivery fraction improves and the end-toend delay for route identification decreases.

\subsection{POSITION-BASED PSO}

Once the candidate nodes are identified, routing must be performed, which is one of the challenging tasks in VANET. Therefore, it becomes difficult to identify an optimal combination for the quality of service in VANET. By applying Particle Swarm Optimization, optimal routing in VANET is easily measured. However, the control parameters in PSO play a major role with the slight differences in the control parameter that results in different performances. Hence, the PPSO-FL, Position-based PSO is designed to minimize the dependency of the control parameters and therefore improve the efficiency of routing in VANET.
Let us consider the (particles) candidate nodes ' $C N$ ' as a set of random solutions through which the source node ' $v_{i}^{\prime}$ 'sends the data packets ' $D P_{i}^{\prime}$ ' and finally reaches the destination node ' $v_{n}$. Each candidate node ' $c n_{i}$ ' is considered to be a potential solution (route) that moves in the search space (i.e. network with transmission range ' $R$ ') following the optimal candidate node.

Each candidate is made up of two ' $n$ ' dimensional vectors, with ' $n$ ' representing the entire network's dimensionality, called the position vector ' $p_{i}^{\prime}$ ' and velocity vector ' $v{ }_{i}^{\prime}$ are expressed as given below.

$$
\begin{aligned}
& p v_{i}=\left(p v_{i 1}, p v_{i 2} \ldots, p v_{i n}\right) \\
& v v_{i}=\left(v v_{i 1}, v v_{i 2}, \ldots, v v_{i n}\right)
\end{aligned}
$$

Where ' $n$ ' corresponds to the candidates in the network.

$$
\begin{gathered}
v v_{i}(T+1)=\omega * v v_{i}(T)+a c_{1} * r n_{1} *\left(P_{\text {best }}-\right. \\
\left.p v_{i}(T)\right)+a c_{2} * r n_{2} *\left(G_{b e s t}-p v_{i}(T)\right) \\
p v_{i}(T+1)=p v_{i}(T)+v v_{i}(T+1)
\end{gathered}
$$

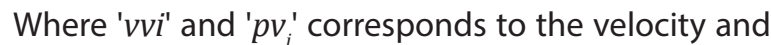
position vector for the 'ith' candidate node in the network. In (11), ' $P_{\text {best }}$ ' corresponds to the previous best candidate node whereas, 'G_best' represents the global best candidate node in the network with ' $r n_{1}$ ' and ' $r n_{2}{ }^{\prime}$ corresponding to the random numbers. Inertial weight is denoted as ' $\omega$ ' whereas, ' $a c_{1}$ ' and ' $a c_{2}$ ' represent the acceleration coefficient values.

$$
\lim _{T \rightarrow \infty}\left(p v_{i}(T)\right)=\frac{a c_{1} * P_{\text {best }}+a c_{2} * G_{\text {best }}}{a c_{1}+a c_{2}}
$$

Where the position of ' $P_{\text {best }}$ ' and ' $G_{\text {best }}$ ' is expressed in (13). Based on the convergence characteristic of PSO, the PPSO-FL uses Position-based PSO (PPSO), each particle (i.e. candidate nodes) only has a position vector and eliminates the velocity vector. Therefore, PPSO in the PPSO-FL eliminates the parameters, ' $\omega, a c_{1}$ and $a c_{2}$ ', minimizing the execution time for routing. The updated positional value based on the PPSO-FL using PPSO is expressed as given below.

$$
\begin{gathered}
p v_{i}(T)=M *\left(\frac{G_{\text {best }}+P_{\text {best }}}{2},\left|G_{\text {best }}, P_{\text {best }}\right|\right) \\
p v_{i}(T)=M *\left(\mu\left(G_{\text {best }}, P_{\text {best }}\right), \sigma\left(G_{\text {best }}, P_{\text {best }}\right)\right)
\end{gathered}
$$

Where ' $M\left(^{*}\right)^{\prime}$ corresponds to the updated positional value based on the average ' $\left(G_{\text {best }}+P_{\text {best }}\right) / 2$ ' and standard deviation ' $/ G_{\text {best }} P_{\text {best }} /$ respectively. Let us consider a source node ' $S$ ', candidate nodes ' $v_{1}, v_{2^{2}}, v_{3^{\prime}}, v_{4}, v_{5^{\prime}}, v_{6}, v_{7}, v_{8^{\prime}}$ $v_{9}, v_{10^{\prime}} v_{11}^{\prime}$ 'through which the data packets ' $D P_{i}^{\prime}$ ' has to be sent to the destination node ' $D$ ' respectively. Fig. 5 shows the construction of the optimized routing model using PPSO with three possible routes identified.

As shown in Fig. 5, three routes exist between the source node ' $S$ ' and destination node 'D'. They are (i) route 1: 'S $\mathrm{Cn_{1 }} \rightarrow \mathrm{Cn}_{2} \rightarrow \mathrm{Cn}_{8} \rightarrow D^{\prime}$ ', (ii) route $2:$ ' $S \rightarrow \mathrm{Cn}_{3} \rightarrow$

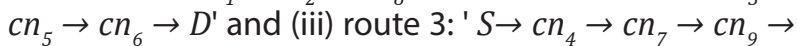
$\mathrm{Cn}_{10} \rightarrow \mathrm{Cn}_{11} \rightarrow D^{\prime}$ respectively. By applying the proposed PPSO, the optimal route is 'S $\rightarrow \mathrm{Cn}_{3} \rightarrow \mathrm{Cn}_{5} \rightarrow \mathrm{Cn}_{6} \rightarrow D$ '. 


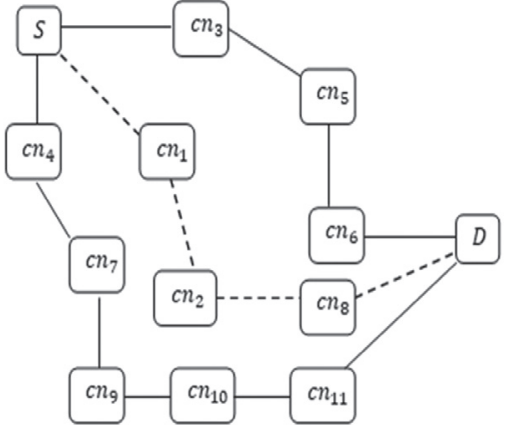

Fig. 5 Construction of optimized routing model

Fig. 6 shows the PPSO algorithm, aiming at improving the identification of optimal routes by the candidate nodes, therefore improving the routing efficiency in VANET.

Input: Source node ' $S$ ', Destination node ' $D$ ', candidate nodes' $C N=c n_{1}, C n_{2}, \ldots, c n_{n}$ ', Data Packets' $D P=$ $D P_{1}, D P_{2} \ldots, D P_{n}$ ', Global best ' $G_{\text {best }}$ ', previous best candidate node ' $P_{\text {best }}$ '

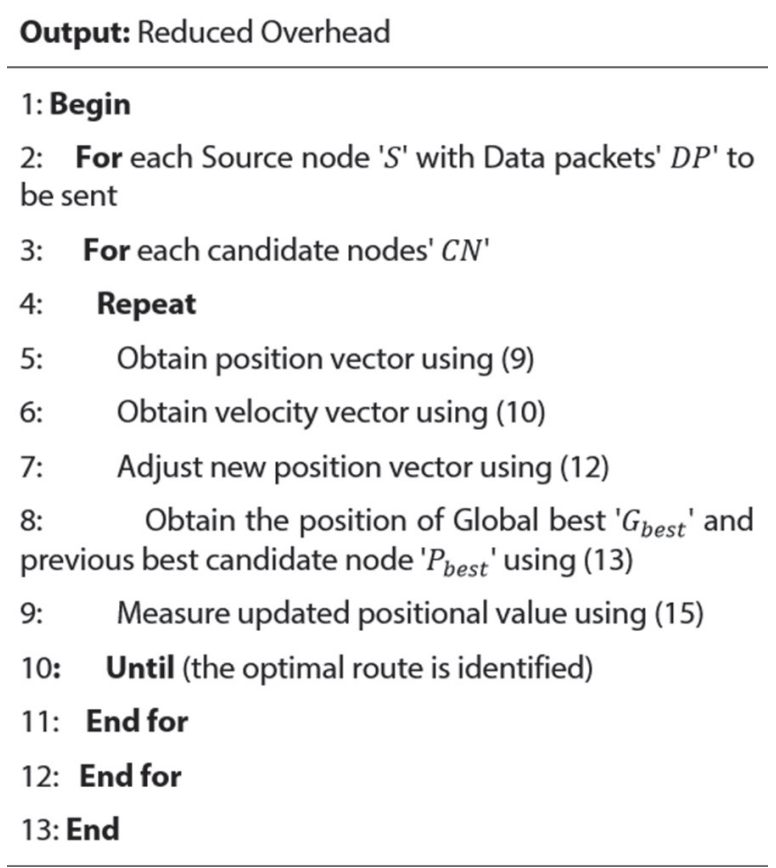

Fig. 6 PPSO Algorithm

In this paper, a position-based particle swarm optimization algorithm by evaluating the candidate nodes is presented. Using the optimal candidate node and randomly adjusting the candidate nodes, the particles' inertia is heterogeneous. The diversity of learning between the particles (i.e. candidate nodes) is increased, and the candidate nodes quickly identify the optimal solution, which in turn paves the way for routing efficiency.

\section{SIMULATION AND RESULTS ANALYSIS}

An extensive simulation has been performed to investigate the proposed PPSO-FL routing optimization. The results outcomes have been compared against the two existing routing algorithms, namely, Virtual Node Ad hoc On-demand Distance Vector (VNAODV) [1] and Long Lifetime Anypaths (LLA) [16].

\subsection{SIMULATION ENVIRONMENT AND PARAMETERS}

NS-2.35 network simulator has been used in the simulation of the PPSO-FL method. NS-2 simulator has been used to create mobility log files with the simulation parameters listed in Table 1. Once the network is deployed with maximum traffic flow based on the simulation parameter listed, the simulation has been performed in different VANET scenarios

Table 1 Simulation parameters

\begin{tabular}{|cc|}
\hline Parameter & Value \\
\hline Node density & $10,20,30,40,50,60,70$ \\
\hline Simulation area & $2000 \mathrm{~m} \times 2000 \mathrm{~m}$ \\
\hline Vehicle speed & $15 \mathrm{~m} / \mathrm{s}$ \\
\hline Transmission range & $300 \mathrm{~m}$ \\
\hline Transmission rate & $4 \mathrm{Mbps}$ \\
\hline Data packet size & $1000 \mathrm{bytes}$ \\
\hline Simulation time & $1400 \mathrm{~s}$ \\
\hline Vehicle speed & $1.4-16.7 \mathrm{~m} / \mathrm{s}$ \\
\hline
\end{tabular}

\subsection{PERFORMANCE METRICS}

Packet Delivery Fraction (PDF): routing efficiency is defined as the total number of data packets sent into the network for every successfully delivered data packet. In other words, the PDF, a measure for routing efficiency, is the ratio of total data packets received over total data packets sent by the source during the simulation period. PDF characterizes the correctness of the routing optimality.

$$
P D F=\left(\frac{D P_{r}}{D P_{S}}\right) * 100
$$

Where the 'PDF' is obtained using the data packets received ' $D P_{r}$ ' and sent ' $D P_{s}$ '.

End-to-end delay: The average end-to-end delay is the average time needed to transfer a data packet from the source vehicle (i.e. node) to the destination vehicle. The lower is the end-to-end delay, the better the application performs.

$$
\operatorname{ETED}=\frac{\operatorname{Time}\left(D P_{r}\right)-\operatorname{Time}\left(D P_{S}\right)}{\left(D P_{r}\right)}
$$

Execution time during routing: The execution time for routing is the time taken for obtaining a route path concerning different routes in the network.

$$
E T=\sum_{i=1}^{n} R_{i} * \text { Time }\left(R_{i}\right)
$$

Where the execution time ' $E T$ ' is measured using the number of routes' $R_{i}^{\prime}$ identified to the time taken for routing 'Time $(R)$ '. 


\subsection{RESULT ANALYSIS}

The results in Table 2 show that the proposed PPSOFL method outperforms the two existing methods VNAODV [1] and LLA [16] in terms of packet delivery fraction. It is noteworthy that the proposed PPSO-FL method considers collectively coordinated metrics concurrently, whereas the existing methods VNAODV and LLA, consider a single objective.

When the data packets are of equal size, the proposed PPSO-FL method outperforms the compared VNAODV and LLA in packet delivery fraction. Because, collectively coordinated metrics such as bandwidth, node energy, and node mobility in finding candidate nodes in the PPSO-FL result in a higher packet delivery fraction. Due to reactive and any path routing for individual vehicles, the packet delivery fraction of VNAODV and LLA improves with increasing data packets. As a result, PPSO-FL outperforms VNAODV and LLA in candidate node selection and packet delivery fraction. The result shows that the PPSO-FL method maximises the packet delivery fraction by $9.84 \%$ more than VNAODV and $23.92 \%$ more than LLA.

Table 2 Performance analysis of packet delivery fraction

\begin{tabular}{cccc} 
Data Packets & \multicolumn{3}{c}{ Packet Delivery Fraction (\%) } \\
& PPSO-FL & VNAODV & LLA \\
\hline 8 & 89.35 & 78.29 & 65.32 \\
\hline 16 & 91.48 & 83.38 & 70.23 \\
\hline 24 & 93.25 & 86.15 & 74.19 \\
\hline 32 & 88.49 & 78.39 & 65.28 \\
\hline 40 & 90.21 & 81.11 & 68.37 \\
\hline 48 & 93.17 & 85.07 & 72.14 \\
\hline
\end{tabular}
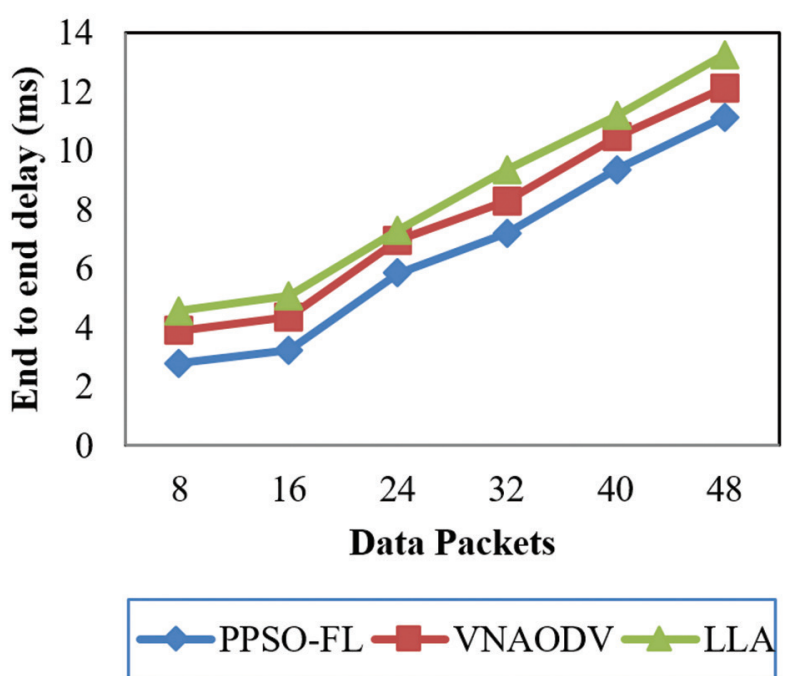

Fig. 7 Measure of end to end delay

Fig. 7 shows the end-to-end delay for different packets transmitted by 60 different nodes. When the speed and density of the data packet increase, the frequency topology changes also increase. Frequent route reconstruc- tion increases control overhead, potentially increasing congestion rate and packet collisions. Fig. 7 shows that the PPSO-FL method effectively reduces end-to-end delay, particularly when the speed is high, and the size of data packets is small. The topology changes fast as the speed increases, and thus the size of data packets increases, increasing the end-to-end delay. However, compared to the existing VNAODV and LLA, PPSO-FL shows significant improvements. The VNAODV and LLA fail to incorporate the rate of bandwidth, node energy, and mobility in route selection, resulting in this improvement. They provide longer delays if the size of the data packet is large. The fuzzy-based candidate selection method is used in the PPSO-FL method to select the candidate nodes, reducing route rediscovery. As a result, the PPSO-FL method produces the lowest end-to-end delay by $21.34 \%$ compared to VNAODV and $35.60 \%$ compared to $L L A$, respectively.

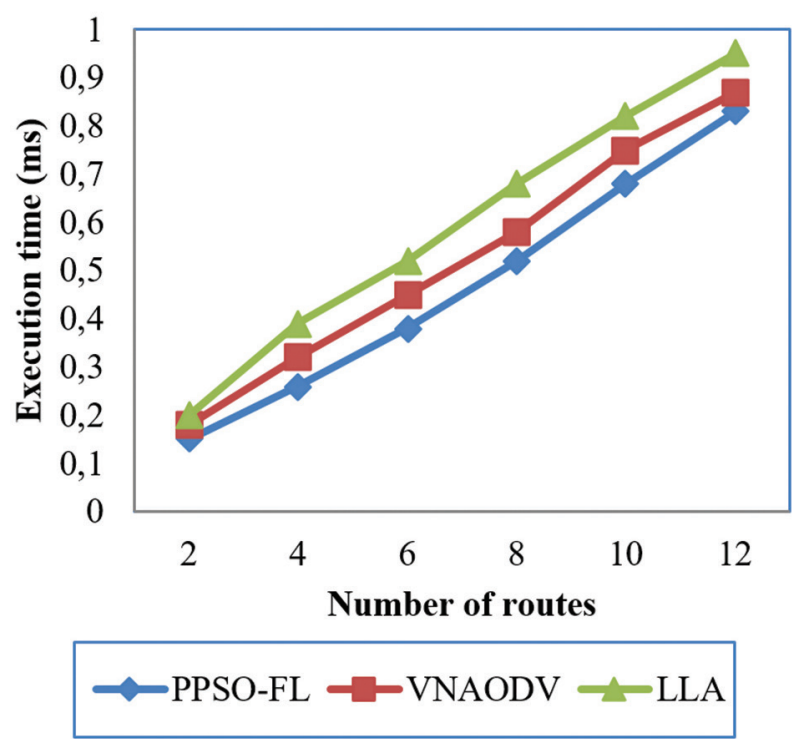

Fig. 8 Measure of execution time

In Fig. 8, we evaluate the execution time for routing using the three methods PPSO-FL, VNAODV, and LLA that presents the relationship between execution time and the number of routes identified. The results proved that packets routed through PPSO-FL require fewer candidate nodes to reach a destination than state-ofthe-art routing protocols. The Position-based PSO that eliminates the dependence of parameters, ' $\omega, a c_{1}$ and $a c_{2}{ }^{\prime}$ with direction toward the destination in-network, results in better performance, reduces the execution time for routing using PPSO-FL by $14.69 \%$ compared to VNAODV and $30.99 \%$ compared to LLA.

\section{CONCLUSION}

In this paper, we have designed a Position Particle Swarm Optimization with Fuzzy Logic (PPSO-FL) method for VANETs, leveraging the collectively coordinated metrics instead of instantaneous velocity. Furthermore, when determining the candidate node, the proposed 
method takes into account the maximum collective coordinated outcome. A practical solution is provided utilising position-based PSO based on the approach, ensuring optimal routing based on the converging characteristic of PSO. Because, packet delivery fraction is the essential element impacting routing efficiency of all parameters, precisely calculating this value is critical. We used updated positional values to remove the control coefficients, resulting in optimal routing that avoided the effects of rapid velocity changes. The simulation results show that, when compared to previous research, the suggested algorithm increases the packet delivery fraction for various numbers of routes and packets. The proposed model has a better performance in terms of execution time and end-to-end delay.

\section{REFERENCES:}

[1] J. F. Bravo-Torres, M. López-Nores, Y. Blanco-Fernández, J.J Pazos-Arias, M. Ramos-Cabrer, A. Gil-Solla, "Optimizing Reactive Routing Over Virtual Nodes in VANETs," IEEE Transactions on Vehicular Technology, Vol. 65, No. 4, 2016, pp. 2274-2294.

[2] H. Dang, H. Wu, "Clustering and cluster-based routing protocol for delay-tolerant mobile networks", IEEE Transactions on Wireless Communications, Vol. 9, No. 6, 2010, pp. 1874-1881.

[3] F. De Rango, F. Guerriero, P. Fazio," Link-stability and energy aware routing protocol in distributed wireless networks", IEEE Transactions on Parallel and Distributed Systems, Vol. 23, No. 4, 2010, pp. 713-726.

[4] M. E. A Fekair, A. Lakas, A. Korichi, N. Lagraa, "An efficient fuzzy logic-based and bio-inspired QoScompliant routing scheme for VANET", International Journal of Embedded Systems, Vol. 11, No. 1, 2019, pp. 46-59.

[5] I. W. H. Ho, K. K. Leung, J. W. Polak, "Stochastic model and connectivity dynamics for VANETs in signalized road systems", IEEE/ACM Transactions on Networking, Vol. 19, No. 1, 2010, pp. 195-208.

[6] Hu, T., \& Fei, Y. QELAR: "A machine-learning-based adaptive routing protocol for energy-efficient and lifetime-extended underwater sensor networks", IEEE Transactions on Mobile Computing, Vol. 9, No. 6, 2010, pp. 796-809.

[7] J. Ni, H. Xie, S. Tatikonda and Y. R. Yang, "Efficient and Dynamic Routing Topology Inference from Endto-End Measurements", IEEE/ACM Transactions on Networking, Vol. 18, No. 1, 2010, pp. 123-135.
[8] J. Jeong, S. Guo, Y. Gu, T. He, D. Du, "Trajectory-based statistical forwarding for multihop infrastructure-tovehicle data delivery", IEEE Transactions on Mobile Computing, Vol. 11, No. 10, 2011, pp. 1523-1537.

[9] R. Kaushik, Chowdhury, Marco Di Felice, Luciano Bononi, "XCHARM: A routing protocol for multichannel wireless mesh networks", Computer Communications, Vol. 36, No. 14, 2013, pp. 1485-1497.

[10] J. Kennedy, "Bare bones particle swarms", Proceedings of the IEEE Swarm Intelligence Symposium, Indianapolis, IN, USA, 26-26 April 2003, pp. 80-87.

[11] S. S. Lam, C. Qian, "Geographic routing in d-dimensional spaces with guaranteed delivery and low stretch. ACM SIGMETRICS Performance Evaluation Review", Vol. 39, No. 1, 2011, pp. 217-228.

[12] Y. Mao, F. Wang, L. Qiu, S. Lam, J. Smith, "S4: Small state and small stretch compact routing protocol for large static wireless networks", IEEE/ACM Transactions on Networking, Vol. 18, No. 3, 2010, pp. 761-774.

[13] K. Mershad, H. Artail, "A framework for secure and efficient data acquisition in vehicular ad hoc networks", IEEE Transactions on vehicular technology, Vol. 62, No. 2, 2012, pp. 536-551.

[14] G. Parissidis, M. Karaliopoulos, T. Spyropoulos, B. Plattner, "Interference-aware routing in wireless multihop networks", IEEE Transactions on Mobile Computing, Vol. 10, No. 5, 2010, pp. 716-733.

[15] R. Purkait, S. Tripathi, " Fuzzy Logic Based MultiCriteria Intelligent Forward Routing in VANET", Wireless Personal Communications, Vol. 111, 2020, pp. 1871-1897.

[16] J. Rak, "LLA: A New Anypath Routing Scheme Providing Long Path Lifetime in VANETs", IEEE Communications Letters, Vol. 18, No. 2, pp. 2014281-284.

[17] F. J. Ros, P. M. Ruiz, I.Stojmenovic, "Acknowledgment-Based Broadcast Protocol for Reliable and Efficient Data Dissemination in Vehicular Ad Hoc Networks", IEEE Transactions on Mobile Computing, Vol. 11, No. 1, 2012, pp. 33-46.

[18] S. Wang, A. Basalamah, S. M. Kim, S. Guo, Y. Tobe, T. $\mathrm{He}$, "Link-correlation-aware opportunistic routing in wireless networks", IEEE Transactions on Wireless Communications, Vol. 14, No. 1, 2014, pp. 47-56. 
[19] W. Wang, X. Liu, D. Krishnaswamy, "Robust routing and scheduling in wireless mesh networks under dynamic traffic conditions", IEEE Transactions on Mobile Computing, Vol. 8, No. 12, 2009, pp. 1705-1717.

[20] D. I. N. G Yu, Y. Z. Liu, X. Y. Gong, W. D. Wang, "Road traffic and geography topology based opportunistic routing for VANETs", The Journal of China Universities of Posts and Telecommunications, Vol. 21, No. 4, 2014, pp. 32-39.
[21] J. Yu, Y. Qi, G. Wang, X. Gu, "A cluster-based routing protocol for wireless sensor networks with nonuniform node distribution", AEU-International Journal of Electronics and Communications, Vol. 66, No. 1, 2012, pp. 54-61.

[22] X. M. Zhang, E. B. Wang, J. J. Xia, D. K. Sung, "A neighbor coverage-based probabilistic rebroadcast for reducing routing overhead in mobile ad hoc networks", IEEE transactions on mobile computing, Vol. 12, No. 3, 2012, pp. 424-433. 